\title{
Las golosinas en la alimentación infantil. Análisis antropológico nutricional
}

\author{
Portia Jackson ${ }^{1,2, a}$, Marcela Romo $\mathbf{M}^{1 \mathrm{~b}}$, \\ Marcela Castillo A ${ }^{1 \mathrm{c}}$, Carlos Castillo-Durán' \\ Junk food consumption and child \\ nutrition in Chile
}

The increasing consumption of junk food and snacks in Chile in recent years and its association with marketing strategies and prevalent diseases, is reviewed. In the context of world economy, junk food is a global phenomenon. The availability of junk food and snacks at low prices and marketing has triggered an evolution of consumption of foods that require neither the structure nor the preparation of a formal meal. Many studies have suggested that the increase in snack consumption is associated with an increase in obesity, tooth decay and other chronic diseases among children and adolescents. The hypothesis suggests a link between the pattern of snack consumption and an increase in the energy density of food consumed, a decrease in satiety, passive over consumption, and an increase in obesity. Between 1977 and 1996, the contribution of snacks to daily energy intake among children between 2 and 5 years increased by $30 \%$ in the United States. In each age group in Chile, the frequency of non-transmissible chronic diseases is increasing due primarily to a westernized diet that is high in fat, cholesterol, sodium, and sugar and a sedentary lifestyle. Education about junk food consumption and healthy eating habits in the family, starting since childbirth and public policies about healthy lifestyles should be strengthened (Rev Méd Chile 2004; 132: 1235-42).

(Key Words: Child nutrition; Diet; Snacks)

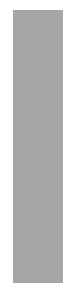

Recibido el 29 de enero, 2004. Aceptado el 16 de junio, 2004.

Trabajo financiado parcialmente por DI SOC-01/12-2, Universidad de Chile y Minority International Research Training Program, Fogarty, University of Michigan.

${ }^{1}$ Instituto de Nutrición y Tecnología de los Alimentos (INTA), Universidad de Chile.

2University of Michigan, School of Public Health.

aAntropóloga, M.PH

bAntropóloga, M.Sc

cPsicóloga, M-Phil

Correspondencia a: Carlos Castillo D. INTA, Universidad de Chile. Macul 5540. Fax 562-2214030. Santiago, Chile.

E-mail: ccastd@inta.cl 
$E^{n}$ los últimos años ha aumentado la preocupa ción acerca del rol que las golosinas juegan en la alimentación infantil. Estudios recientes utilizan diferentes terminologías para el concepto golosinas; en este trabajo se entiende por golosinas aquellos alimentos industriales, nutricionalmente desbalanceados y con un alto contenido de hidratos de carbono, grasas o sal. Algunos estudios incluyen golosinas y otro tipo de ingredientes y alimentos bajo los conceptos de "densos en energía y pobres en nutrientes"1,2 o "altos en calorías y de baja densidad de nutrientes" 3 o "alimentos que contribuyen a la ingesta energética"4 (Figura 1). Se enfatiza el desbalance de estos alimentos, así como en la categoría "comida chatarra" concepto que, además, los relaciona con una categonía de alimentos percibidos socialmente como no saludables ${ }^{5}$. Otros conceptos asociados a golosinas utilizan criterios como el lugar o el horario en que son consumidos. Por ejemplo, "snacks" o "colación" se ha definido como alimentos consumidos fuera del horario de comida formal, alimentos comidos en un período de quince minutos o menos y tipos de comida específicos, en su mayoría golosinas ${ }^{6,7}$.
Un concepto a veces asimilado al de golosina es el de "comida rápida" el que se refiere a alimentos expendidos por empresas comerciales, semilistos para consumir, bajo formas de preparación estándares y basados en ingredientes como hamburguesas, pollo, pescado, pizza, sandwich etc $^{8}$. Estas últimas preparaciones no son necesariamente desbalanceadas, nutricionalmente.

Desde una perspectiva antropológica, el concepto de golosina es el de un producto preferentemente infantil, consumido fuera de los horarios de comida regulares y que muchas veces no es clasificado como un alimento de acuerdo a las conceptualizaciones populares.

No obstante, la asociación más potente de este concepto es con el placer, ya que como lo define la Real Academia Española, golosina es un "manjar delicado, generalmente dulce, que sirve más para el gusto que para el sustento", la que a su vez define placer como "satisfacción, sensación agradable producida por la realización o suscepción de algo que gusta o complace" ${ }^{\prime \prime}$. El placer asociado con la comida tiene una larga historia; algunas de las actuales actitudes morales y éticas hacia la

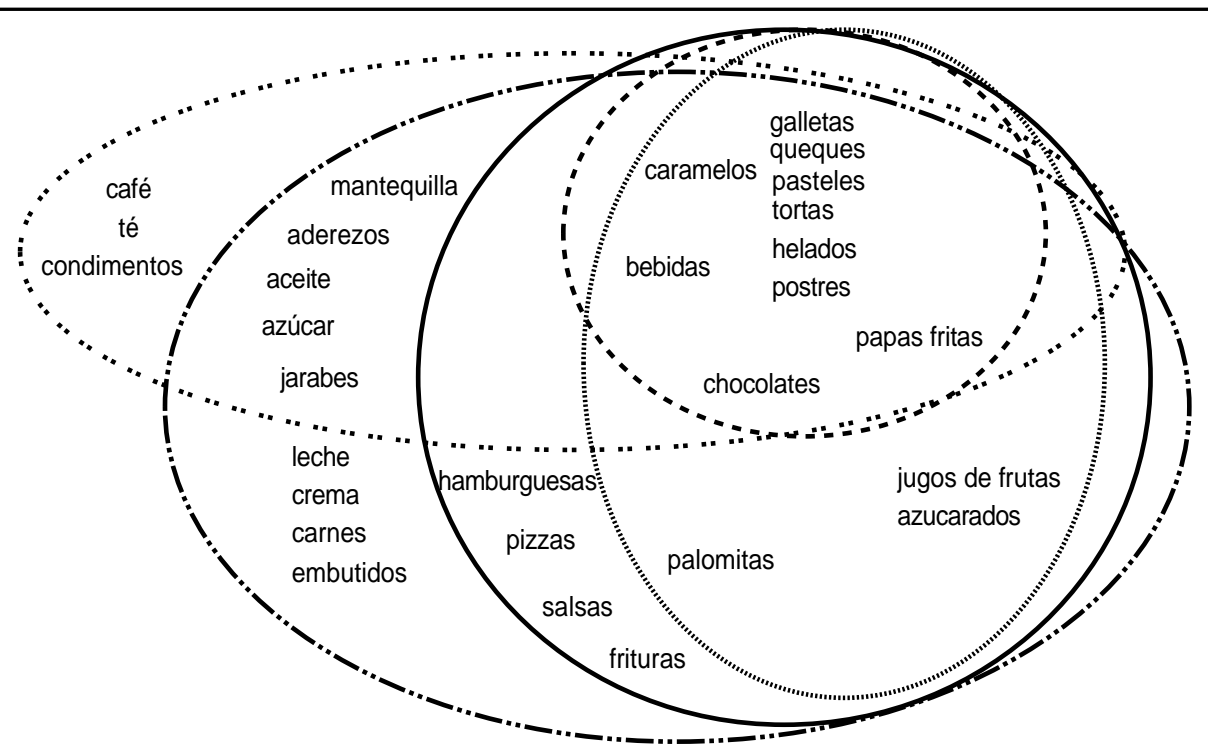

....... Densos en energía y pobres en nutrientes (energy-dense and nutrient-poor foods).

- - - - Altos en calorías y baja densidad de nutrientes (high-calorie, low-nutrient-dense).

-..... Alimentos que contribuyen a la ingesta energética.

..................... Snacks.

Comida chatarra (junk food).

FIGURA 1. Conceptos utilizados en investigaciones asociados a golosinas. 
comida provienen del pensamiento estoico de la filosofía griega, quienes manifestaron preocupación por la idea de moderar la conducta hacia la comida como muestra de autocontrol. Hasta hoy, el placer del comer es visto como pecaminoso y autoindulgente. Muchos alimentos, especialmente las golosinas, son incluso publicitados de esta forma; se enfatizan los aspectos sensoriales, sociales y emocionales que dan placer al consumir el producto.

Hay muchos elementos en la comida que producen placer y éste no se reduce al acto mismo de ingerir un determinado alimento; están también el goce de compartir la comida, el placer de cocinar para los que se quiere, mostrándoles así afecto y también el placer obtenido en la adquisición de la comida a través de actividades colectivas como la cosecha, la pesca o la compra.

Algunos estudios sugieren que las emociones intensamente placenteras, asociadas a la comida están acompañadas por actividad en el sistema neurológico en la base de los procesos de recompensa/motivación, emocionales (límbicos) y de alerta. La experiencia placentera que acompaña la ingesta de chocolate se ha visto correlacionada con actividad en el mesencéfalo, ínsula, la región subcallosa y la corteza órbito frontal ${ }^{10}$.

Una publicación reciente muestra, en estudios con animales de laboratorio, que la grasa y el azúcar son poderosas fuentes de recompensa neurobiológica. Los alimentos que son densos en energía proveen más goce sensorial y más placer que los otros alimentos. Estudios clínicos sugieren que los alimentos más probables de ser apetecidos son aquellos que contienen grasa, azúcar, o ambas. La preferencia humana por el sabor del azúcar y la grasa es innata 0 adquirida muy tempranamente. Estudios con niños muestran consistentemente que la familiaridad con el sabor dulce y la densidad energética en los primeros años son determinantes en las preferencias de estos alimentos en edades posteriores ${ }^{11}$.

\section{HistoRia DE LAS GOLOSINAS}

Los suflitos, palomitas de maiz (cabritas), pretzels y otros productos semejantes se introdujeron en Estados Unidos de América (EE.UU) y Europa a fines del siglo XIX con recetas familiares para eventos especiales $^{12}$. A principios del siglo XX, con la incorporación de maquinaria y la creación de fábricas, se convirtieron en productos industriales, con un crecimiento notable en la oferta y la demanda mundial.

A mediados del siglo XX, empresas estadounidenses y de otros países industrializados, convirtieron la comida en un elemento potente de globalización, exportando al resto del mundo golosinas dulces o saladas y bebidas gaseosas.

\section{La INDUSTRIA DE LAS Golosinas EN CHILE}

En Chile, la industria de las golosinas ha sido desarrollada por empresas nacionales y transnacionales. Las primeras fábricas de golosinas comienzan a fines del siglo XIX (ej: McKay, Costa, Calaf); sin embargo, el desarrollo de una industria alimentaria de golosinas, propiamente tal, parte en la década 1940-49, con empresas como Coca Cola y Ambrosoli. La modernización de los procesos productivos, la diversificación de los productos y la masificación del consumo comienza a desarmollarse a partir de la década 1980-89, alcanzando su mayor fuerza en estas últimas décadas. Actualmente, muchas empresas nacionales han sido absorbidas por empresas mayores nacionales o multinacionales. Por ejemplo: Hucke y Mckay por Nestlé, Costa y Caricia por Carozzi, Dos en Uno por Arcor; Evercrisp, Barcel y Frito Crac por Frito Lay. En el mercado de las golosinas es muy significativa la presencia de transnacionales como Coca Cola (EE.UU), Nestlé (Suiza), Bresler (EE.UU), Arcor (Argentina), Unilever (EE.UU) y Bimbo (México) cuyos productos lideran el mercado en Chile (Tabla 1).

Chile destaca a nivel mundial por el alto consumo de helados, similar al de países europeos, con 6 litros per cápita anual (Publimark 2001; 148: 6-10) y a nivel latinoamericano en el consumo de bebidas gaseosas, con 90 litros per cápita anual (Diario ESTRATEGIA 23 de septiembre de 2002). De acuerdo a estudios de mercado, los productos con perspectivas de aumentar su consumo en los próximos años son los postres lácteos ${ }^{13}$ y las galletas (Publimark 2002; 155: 52-56).

\section{ESTRATEGIAS EN EL MERCADO DE LAS GOLOSINAS}

Las empresas dedicadas al rubro golosinas enfocan sus productos de acuerdo a la demanda por grupos de edad, siendo los niños y los adolescen- 
Tabla 1. Principales empresas productoras, marcas y tipos de golosinas en Chile (2003)

\begin{tabular}{|lcccccc|}
\hline $\begin{array}{l}\text { Empresas productoras y } \\
\text { marcas }\end{array}$ & $\begin{array}{c}\text { Producto } \\
\text { procesado } \\
\text { salado }\end{array}$ & $\begin{array}{c}\text { Producto } \\
\text { procesado } \\
\text { dulce }\end{array}$ & $\begin{array}{c}\text { Bebida, } \\
\text { jugo y } \\
\text { helado }\end{array}$ & $\begin{array}{c}\text { Caramelo, } \\
\text { caluga y } \\
\text { chocolate }\end{array}$ & $\begin{array}{c}\text { Postre } \\
\text { (jalea, } \\
\text { flan) }\end{array}$ & $\begin{array}{c}\text { Tipos de } \\
\text { Productos } \\
\text { Total }\end{array}$ \\
\hline Carozzi (Costa, Caricia, Ambrosoli) & $\mathrm{X}$ & $\mathrm{X}$ & $\mathrm{X}$ & $\mathrm{X}$ & $\mathrm{X}$ & 5 \\
Nestlé (Savory, McKay) & & $\mathrm{X}$ & $\mathrm{X}$ & $\mathrm{X}$ & $\mathrm{X}$ & 4 \\
Parmalat & $\mathrm{X}$ & $\mathrm{X}$ & $\mathrm{X}$ & & $\mathrm{X}$ & 3 \\
Arcor (Dos en Uno) & $\mathrm{X}$ & $\mathrm{X}$ & & $\mathrm{X}$ & & 3 \\
Bimbo (Ideal, Marinela) & $\mathrm{X}$ & $\mathrm{X}$ & & $\mathrm{X}$ & & 2 \\
Lucchetti (Calaf) & & $\mathrm{X}$ & $\mathrm{X}$ & $\mathrm{X}$ & & 2 \\
Lay (Evercrisp, Barcel, Frito Crac) & & & $\mathrm{X}$ & & $\mathrm{X}$ & 2 \\
Trendy (Panda) & & & $\mathrm{X}$ & & & 2 \\
Loncoleche & & & $\mathrm{X}$ & & & 1 \\
Cocacola (Kapo, Andifrut) & & $\mathrm{X}$ & & & 1 \\
Ecusa (Bilz y Pap, Pepsi, Watts) & & & $\mathrm{X}$ & & $\mathrm{X}$ & 1 \\
Corpora (Zuco, Yupi, Yuz) & & & & & & 1 \\
Bresler, Chamonix & & & & & \\
Soprole & & & & & & \\
\hline
\end{tabular}

Fuente: Construido en base a la información de las páginas web de las empresas.

tes dos de sus mercados más importantes. Los productos diseñados para niños se caracterizan por un precio económico y de «compra impulsiva» (el cliente tiene un par de segundos para evaluar y decidir por el tipo de producto, presentación, calidad y rango de precios). Además, éstos van en envases especiales o regalos (ej: juegos, figuras, tatuajes). Para los adolescentes los productos son más grandes, pueden aumentar algo en precio y van asociados a campañas promocionales, remarcando los conceptos de modernidad y juventud (Capital 2003; 11: 68-72).

Estos productos están diseñados, principalmente, para la venta callejera (ej: microbús, carritos, vendedores ambulantes) y pequeños negocios (kioscos y almacenes). Aquellas marcas de precios más baratos no invierten en publicidad ya que su estrategia está basada en su relación precio-calidad. El gran mercado actual está en la unidad monetaria más usada (moneda de 100 pesos chilenos), ya que las monedas fraccionadas no funcionan en el mercado de compra impulsiva.

La competencia en el mercado de las golosinas es muy fuerte ya que no sólo compiten las empresas que desarrollan un mismo tipo de producto, sino que también se compite entre productos distintos: las bebidas con los helados, los chocolates con los productos bañados y rellenos (Publimark 1999; 126: 44-46). Las grandes compañías desarrollan campañas de marketing en los colegios, y lugares de recreación como playas y piscinas. Los niños son un blanco muy importante en esta industria, no sólo por su poder adquisitivo, sino también porque se convierte en la entrada del producto en la familia. Como estrategia de marketing se están diseñando productos atractivos para los niños, que a su vez se presentan con una imagen «nutritiva» para que los padres sientan que están entregando algo más que una golosina (Publimark 2001; 141: 6-10). También se está usando la asociación con licencias de programas de televisión de alto consumo entre los pequeños (ej: WWF con lucha libre en EE.UU, Bresler - Digimon y Looney Tunes con Trendy (Publimark 2002; 151: 6-10)).

Distintas empresas, a su vez, tienen líneas de productos y marcas diferenciadas de acuerdo al nivel socioeconómico y canales de venta. En los últimos años también se ha observado cómo los envases dirigidos a la familia, hoy tienen su versión para el consumo individual y callejero, especialmente desarrollado en bebidas y galletas. 
Prácticas en el consumo de las golosinas en Chile

Hay dos modalidades principales en el consumo de las golosinas. Primero, en los mismos lugares de adquisición, incluyendo supermercado, almacén, al paso, en centros de comida, y en el colegio (kioscos, triciclos). La otra forma es como colaciones en jardines infantiles, colegio, lugar de trabajo, fiestas y paseos.

Según un estudio reciente efectuado en la comuna de La Pintana, en Santiago la incorporación de golosinas en la alimentación de niños ocurre ya a partir del segundo semestre de la vida y en especial durante el segundo año (Castillo, comunicación preliminar). Un estudio en escolares de $5^{0}$ a $8^{0}$ básico, mostró que cerca de la mitad de las colaciones que los niños llevaban al colegio eran golosinas y entre aquellos que compraban algún alimento 95\% adquiría golosinas y gaseosas $^{14}$. De acuerdo al mismo estudio, el consumo promedio diario de snacks alcanzó ente 324 y 424 calorías en las distintas localidades estudiadas ${ }^{15}$.

\section{VISIÓN ANTROPOLÓGICA DE LAS GOLOSINAS}

Para comprender los factores que están incidiendo en la alimentación de los niños chilenos, es importante una visión desde la antropología nutricional. Por ejemplo, al examinar cómo se define lo que es una comida o la que es una colación, las personas en general no consideran las golosinas 0 las bebidas gaseosas como alimentos, por lo que no se contabilizan al pensar en la ingesta diaria.

A la luz de la información recopilada y siguiendo el modelo explicativo de los componentes del proceso de elección de alimentos ${ }^{16}$ se puede decir que en los individuos, en especial en los niños, enfrentados a la decisión de elegir entre la variada oferta de golosinas, priman las percepciones sensoriales y en particular el placer. La alta palatabilidad de las golosinas es la característica más importante en su elección (Figura 2).

El precio, la conveniencia y las relaciones sociales, son otros valores considerados al momento de elegir un alimento; la industria se ha

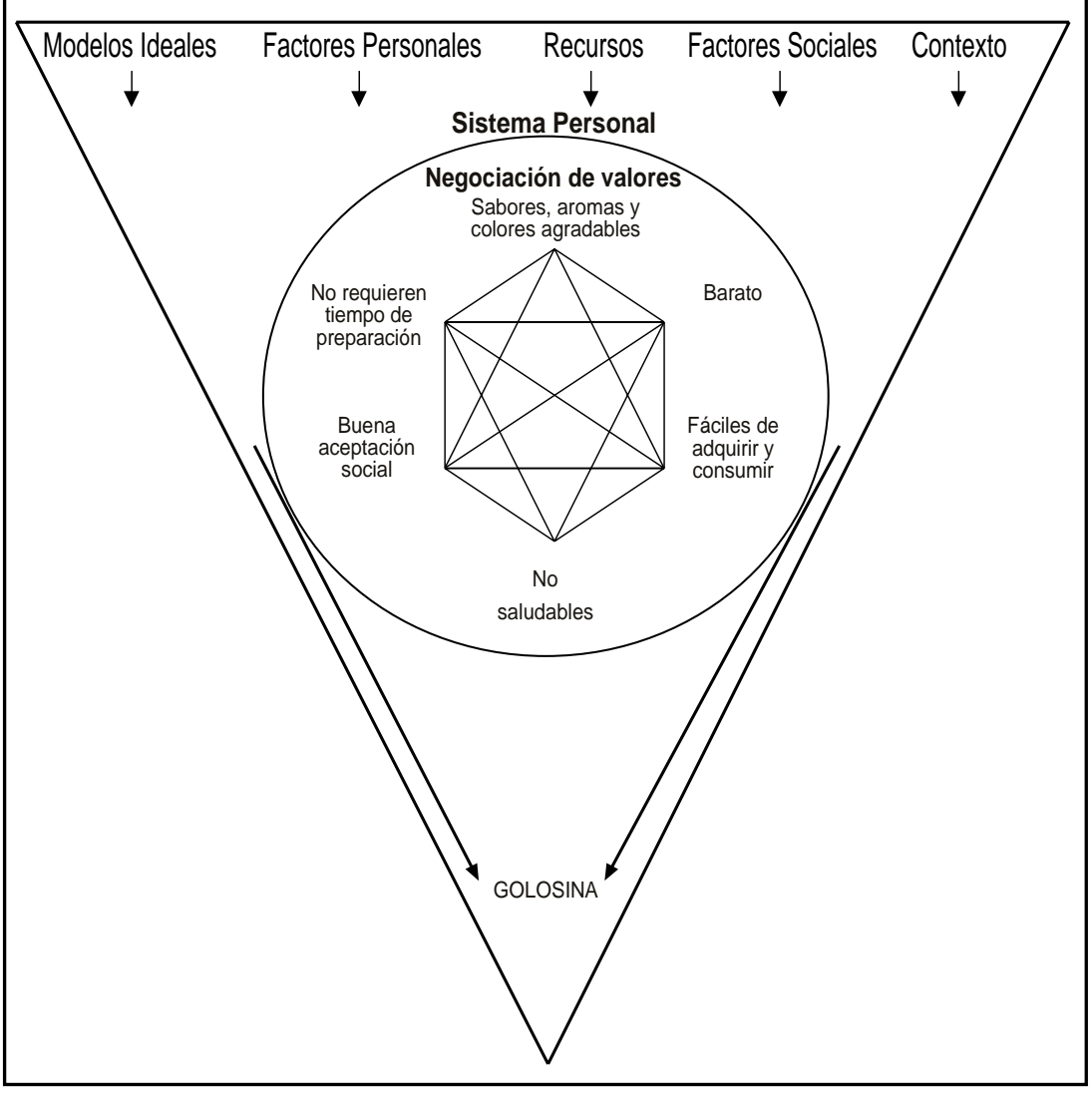

Figura 2. Factores que influyen en la decisión de comer golosinas. Adaptados de Furst et $\mathrm{al}^{16}, \ll$ conceptual model of the components in the food choice process». 
preocupado de estos componentes al ofrecer golosinas a bajo costo, fáciles de adquirir y consumir, y de gran aceptabilidad social en términos que los niños requieren consumirlas para insertarse dentro del grupo de pares. El componente valórico de la salud queda reducido a un segundo plano, aunque la industria ha comenzado a incorporar conceptos nutricionales como la suplementación con nutrientes específicos.

Entre los factores que modelan la elección de golosinas tenemos los modelos de alimentación transmitidos por la escuela; los equipos médicos y los medios de comunicación; factores personales tales como educación, percepciones sensoriales y estado de salud; los recursos económicos; la red social a la que se pertenece; y el contexto en el que se produce la situación de alimentación. La sociedad, en general, empuja hacia una actitud que privilegia el placer y el consumo por sobre la salud. Esto ha llevado a una descontextualización del consumo de golosinas que pasaron de ser un alimento consumido en ocasiones o lugares especiales, a un alimento de consumo habitual o diario. Otros factores importantes en esta evolución son la decreciente disponibilidad de tiempo de la madre para preparar comidas habituales 0 colaciones y la asistencia del niño a jardín infantil, donde estos tipos de alimentos son introducidos en colaciones y celebraciones.

Hoy se valora la autonomía e independencia temprana de los niños, se les da dinero precozmente, teniendo la posibilidad de tomar decisiones en su alimentación. Yáñez et al, observaron que casi la totalidad de los niños estudiados disponía de dinero «en alguna ocasión»y 34\% «siempre»disponía de éste ${ }^{15}$.

Otro factor cultural que incide en la definición de una dieta saludable es la percepción de la imagen corporal apropiada o saludable. En el caso de los niños pequeños se prefieren los niños 〈gorditos»como signo de salud o protección frente a eventuales enfermedades. De acuerdo al estudio de Díaz ${ }^{17}, 37,5 \%$ de las madres pensaban que su niño obeso tenía un poco de sobrepeso y $26,6 \%$ creía que su niño obeso tenía peso normal.

\section{IMPACTO DE LAS GOLOSINAS EN LA SALUD, TENDENCIAS}

En las últimas dos décadas Chile ha pasado de ser un país con alta tasa de desnutrición y baja tasa de obesidad, a una escasa desnutrición y altas tasas de obesidad en todos los grupos etarios ${ }^{18}$. En dos investigaciones realizadas en 1988 y 1992 en adultos de Santiago, la obesidad aumentó de 6 a $11 \%$ en los hombres y de 14 a $24 \%$ en mujeres. La prevalencia de obesidad infantil se ha duplicado en los últimos 10 años, alcanzando 17,2\% el año 2002 entre los niños de 6 años ${ }^{19}$. El fenómeno es global, ya que en EE.UU la obesidad en la población adulta alcanza $30,5 \% 20$, mientras que el sobrepeso en los niños de 6 a 11 años es de 15,3\%.

Es aún discutido el impacto de las golosinas en la salud de niños y adolescentes. Existen estudios que avalan la hipótesis de una relación entre el incremento en el consumo de golosinas y colaciones y el incremento en la densidad total de energía consumida, tanto en adultos como en niños ${ }^{6}$. Se propone una conexión entre los patrones de consumo de snacks, el incremento en la densidad de energía de los alimentos consumidos y una alteración de la sensación de saciedad, con un sobreconsumo pasivo de energía y un incremento en la obesidad ${ }^{7}$. Sin embargo, estudios como el de Bandini et $\mathrm{al}^{3}$ no logran establecer diferencias en el consumo de golosinas entre grupos de obesos y no obesos. Esto puede deberse a las dificultades metodológicas para determinar la ingesta de este tipo de alimentos ya que muchas veces no son reportados ${ }^{21}$.

Comparados con las comidas normales, las golosinas incrementan la densidad energética y la proporción de energía proveniente de la grasa e hidratos de carbono, mientras que son deficientes en otros nutrientes 6,7 .

En los últimos 50 años, la tasa de consumo de bebidas gaseosas en Estados Unidos ha aumentado en $500 \%$ per cápita 22 , siendo la principal fuente de azúcar extra en la dieta de adolescentes ${ }^{23,24}$. Se ha demostrado que el consumo de bebidas en niños está asociado con un aumento en la obesidad ${ }^{25}$; se observa un reemplazo de la leche en la dieta resultando en déficit de calcio ${ }^{7} \mathrm{y}$ un aumento en las caries dentales asociado también al consumo de snacks dulces ${ }^{22,26}$. Existe una hipótesis que plantea que el consumo de carbohidratos en forma líquida no compensa la energía consumida produciendo un balance energético positivo $^{27}$. Se hace imprescindible una educación que enseñe a comer más cereales, frutas $\mathrm{y}$ verduras $^{28}$. 
Concluyendo, como hace un par de décadas dijo el antropólogo norteamericano Marvin Harris $^{29}$, lo bueno para comer se transforma en lo bueno para vender, a despecho de su capacidad nutricional. Se piensa que se tiene poder de decisión sobre lo que se come o se da a los hijos, sin embargo, es la industria la que está decidiendo qué es lo que quiere vender, y su producción está en función de aquello que les genere mayores ganancias. Se ha llegado a la paradoja, en el caso de las golosinas que traen aparejadas un alto costo del envase en comparación con el valor del contenido, que en vez de comprar alimentación se está comprando entretención.

La obesidad debiera enfocarse desde una perspectiva de salud pública, con políticas en los temas de nutrición, medicina y educación, y un abordaje intersectorial, multidisciplinario y preventivo. Es importante, tal como lo señala Busdiecker et al, que las estrategias preventivas para atacar estos problemas comiencen en los primeros años de vida, tomando en cuenta el efecto de la cultura en la formación de prácticas alimentarias ${ }^{30}$.

Los países nórdicos han tenido éxito en la modificación de los hábitos de consumo de su población a través de políticas públicas que han apuntado al incentivo del consumo de alimentos

\section{REFERENCIAS}

1. KaNT AK. Consumption of energy-dense, nutrient poor foods by adult Americans: nutritional and health implications. The Third National Health and Nutrition Examination Survey, 1988-1994. Am J Clin Nutr 2000; 72: 929-36.

2. Newby PK, Peterson K, Berkey C, Leppert J, Wimett WC, CoLdiTz GA. Dietary composition and weight change among low-income preschool children. Arch Pediatr Adolesc Med 2003; 157: 759-64.

3. Bandini LG, Vd D, Must A, Cyr H, Goldberg A, DIETZ WH. Comparison of high-calorie, low-nutrient-dense food consumption among obese and non-obese adolescents. Obes Res 1999; 7: 438-43.

4. Nielsen SI, Siega-Riz AM, Popkin BM. Trends in food locations and sources among adolescents and young adults. Prev Med 2002; 35: 107-13.

5. North K, Emmett P. Multivariate analysis of diet among three-years-old children and associations saludables a través de subsidios o impuestos a determinados productos, a políticas de fomento de la producción y venta de estos alimentos y campañas de educación nutricional ${ }^{31}$. En países industrializados como EE.UU, Australia e Inglaterra se están discutiendo en la actualidad normativas como restricciones a la publicidad sobre comida chatarra, impuestos especiales a estos alimentos, o la prohibición de su venta en las escuelas $^{27,32}$. En Chile también se han tomando iniciativas que apuntan en esta dirección como programas de educación en alimentación saludable para escolares y preescolares ${ }^{33}$ y programas de información al consumidor ${ }^{34}$.

En este sentido es importante dar el impulso a acciones destinadas al apoyo de la producción, comercialización y publicidad de alimentos saludables, de manera que puedan competir con las golosinas. Son necesarias intervenciones educativas tempranas para informar a los padres acerca de las golosinas y colaciones y su composición nutritiva. También es importante discutir, por un lado, el rol de los medios de comunicación en los patrones alimentarios de la población, en especial el impacto que tienen en los niños y por otro lado la toma de conciencia de la influencia que tienen los niños en la decisión de compra y en el consumo familiar de estos productos.

with socio-demographic characteristics. Eur J Clin Nutr 2000; 54: 73-80.

6. Zizza C, Siega-Rzz AM, Popkin BM. Significant increase in young adults' snacking between 19771978 and 1994-1996 represents a cause for concern. Prev Med 2001; 32: 303-10.

7. Jahns L, Siega-Riz AM, Popkin BM. The increasing prevalence of snacking among US children from 1977 to 1996. J Pediatr 2001; 138: 493-8.

8. Newby PK, Muler D, Haufrisch J, Qiao N, Andres $\mathrm{R}$, TUCKER KL. Dietary patterns and changes in body mass index and waist circumference in adults. Am J Clin Nutr 2003; 77: 1417-25.

9. Real Academia Española. Diccionario de la Lengua Española. Madrid: Editorial Espasa-Calpe, 1992.

10. Blood AJ, Zatorre RJ. Intensely pleasurable responses to music correlate with activity in brain regions implicated in reward end emotion. Proc Natl Acad Sci USA 2001; 98: 1181823. 
11. Drewnowski A, Specter S. Poverty and obesity: the role of energy density and energy costs. Am J Clin Nutr 2004; 79: 6-16.

12. Snack Food Association. http://www.sfa.org [Consultado el 24 de junio de 2003].

13. Dirven M, Ortega L El complejo productivo lácteo en Chile. En: Dirven M, editor. Apertura económica y (des)encadenamientos productivos. Reflexiones sobre el complejo lácteo en América Latina. LC/G.2122-P/E Libros de la CEPAL № 61. Santiago: CEPAL, 2001; 143-208.

14. Olivares S, YáñEz R, Díaz N. Publicidad de alimentos y conductas alimentarias en escolares de $5^{\circ}$ a $8^{\circ}$ básico. Rev Chil Nutr 2003; 30: 36-41.

15. Yanez R, Olivares S, Torres I, Guevara M, Díaz N. Consumo de alimentos escolares chilenos. Su relación con las guías y la pirámide alimentaria. Rev Chil Nutr 2001; 28: 422-8.

16. Furst T, Connors M, Bisogni CA, Sobal J, Falk LW. Food choice: a conceptual model of the process. Appetite 1996; 26: 247-65.

17. DíAz M. Percepción materna del estado nutritivo de sus hijos obesos. Rev Chil Pediatr 2000; 71: 316-20.

18. Albala C, Vio F, Kain J, Uauy R. Nutrition transition in Chile: Determinants and consequences. Public Health Nutr 2002; 5: 123-8.

19. JUNAEB. Mapa nutricional de JUNAEB escolares de primero básico, establecimientos municipalizados y particulares subvencionados, según región, comuna y escuela 2002. Departamento Programas Dirección Nacional, JUNAEB, Gobierno de Chile. Disponible en: www.junaeb.cl (Consultado el 22 de diciembre de 2003).

20. Flegal KM, Carroll MD, Ogden CL, Johnson CL. Prevalence and trends in obesity among US adults, 1999-2000. JAMA 2002; 288: 1723-7.

21. Hise ME, Suluv AN DK, Jacobsen DJ, Johnson JL, DONNELL Y JE. Validation of energy intake measurements determined from observer recorded food records and recall methods compared with the doubly labelled water method in overweight and obese individuals. Am J Clin Nutr 2002; 75: 263-7.
22. Touger-Decker R, VAN LOVeren C. Sugars and dental caries. Am J Clin Nutr 2003; 78: 881S-892S.

23. Putnam JJ, Alushouse JE. Food consumption, prices, and expenditures, 1970-97. Washington, DC: Food and Consumers Economics Division, Economic Research Service, US Department of Agriculture, 1999.

24. Guthrie JF, Morton JF. Food sources of added sweeteners in the diets of Americans. J Am Diet Assoc 2000; 100: 43-51.

25. Bowman SA. Diets of individuals based on energy intakes from added sugars. Fam Econ Nutr Rev 1999; 12: 31-8.

26. Ludwig DS, Peterson KE, Gortmaker SL Relation between consumption of sugar-sweetened drinks and childhood obesity: a prospective, observational analysis. Lancet 2001; 357: 505-8.

27. Committee on School Health. Soft drinks in schools. Pediatrics 2004; 113: 152-4.

28. Dimeglo DP, MatTes RD. Liquid versus solid carbohydrate: effects on food intake and body weight. Int J Obes Relat Metab Disord 2000; 24: 794-800.

29. Subar AF, Krebs-Smith SM, Cook A, Kahle LL. Dietary sources of nutrients among US Children, 1989-1991. Pediatrics 1998; 102: 913-23.

30. Harris M. Bueno para comer. Madrid: Alianza Editorial, 1997.

31. Busdiecker S, Castilo C, Salas A. Cambios en los hábitos de alimentación durante la infancia: una visión antropológica. Rev Chil Pediatr 2000; 71: 5-11.

32. Roos G, Lfan M, Anderson A. Dietary interventions in Finland, Norway and Sweden: nutrition policies and strategies. J Hum Nutr Diet 2002; 15: 99-110.

33. HABER D. Wellness general of the United Status: a creative approach to promote family and community health. Fam Commun Health 2002; 25: 71-82.

34. Salinas J, Vio Del RF. Promoción de la Salud en Chile. Rev Chil Nutr; 2002; 29: 164-73.

35. Domper A, Zacarías L, Olivares S, Hertrampf E. Evaluación de un programa de información en nutrición al consumidor. Rev Chil Nutr 2003; 30: 43-51. 